\title{
Curing Cancer with Nanotherapy Continues to be an Elusive Goal
}

\author{
Priyanka Ray
}

Department of Chemical, Biochemical and Environmental Engineering, University of Maryland, Baltimore County, Baltimore MD, 21250, USA

Article Info

\section{Article Notes}

Received: April 06, 2021

Accepted: May 10, 2021

\section{*Correspondence:}

${ }^{*}$ Dr. Priyanka Ray, Department of Chemical, Biochemical and Environmental Engineering, University of Maryland, Baltimore County, Baltimore MD, 21250, USA;

Email: priyanka@umbc.edu.

(c) 2021 Ray P. This article is distributed under the terms of the Creative Commons Attribution 4.0 International License.
Nanotechnology has aimed to address limitations of new drug design by "repackaging" FDA approved drugs without compromising their therapeutic benefits while suppressing undesired side effects suffered by patients undergoing chemotherapy. The major hurdles faced by small molecular drugs in treating cancer include hydrophobicity, rapid hepatic and renal clearance, short circulation time, off target toxicity and drug resistance. Most of these factors are addressed using nano formulations comprising lipids or polymers and simultaneously striving to enhance the therapeutic index of chemotherapeutic drugs.

Exploiting the difference between the tumor microenvironment (TME) and healthy tissues, scientists have developed a range of stimuli responsive nanomaterials, capable of specifically targeting cancer cells with minimal collateral damage. In this regard, acidity $(\mathrm{pH})^{1-3}$, low oxygen pressure (hypoxia) ${ }^{4-6}$, temperature ${ }^{7}, \mathrm{ROS}^{8}$ and enzyme ${ }^{9}$ gradients have been used as triggers. Drug encapsulation in synthetic or bioderived materials has shown promise as drug delivery agents ${ }^{10-32}$. To enhance drug availability, active targeting strategies employing peptides, aptamers and small molecules have also been employed.

Although nanomaterials are widely researched and appreciated for their advantages over conventional therapeutic methods, there are several limitations to their use. These include targeting properties which are usually achieved by conjugating ligands which bind to over-expressed proteins on cancer cells, but there is a high chance of the nanomaterials leading to off target toxicity when these proteins are produced by healthy cells. Targeting rapidly dividing cells is also a strategy of nanomedicine which may be compromised when there are other growing cells in an acidic environment which do not belong to the tumor tissue, such as are found in the GI tract lining or stomach ${ }^{33}$. In terms of fabrication, reproducibility and scaling up are also challenges thus leading to variations in every batch of nanomaterials produced. Their colloidal stability and shelf life are also factors impeding their translational capacity. Finally, the safety of such materials once injected in the body and their ultimate fate remains controversial ${ }^{34}$.

Despite advances made in preclinical trials with nanomaterials, there still looms a large question regarding the immunogenic response of these carriers. Most in vivo studies use immunocompromised animals to grow xenograft tumors wherein the therapeutic efficiency is evaluated in the absence of immunological response. Due to the heterogeneity of the tumors, and the existence of "cold" and "hot" tumors both immunostimulation and suppression strategies have been studied in depth (Figure 1). 


\begin{tabular}{|l|}
\hline \multicolumn{1}{|c|}{$\begin{array}{c}\text { Cancer vaccine design } \\
\text { strategies using } \\
\text { nanomaterials }\end{array}$} \\
Targeting DCs \\
Lymph node drainage \\
Antigen presentation \\
Co-delivery of antigen and \\
adjuvant
\end{tabular}

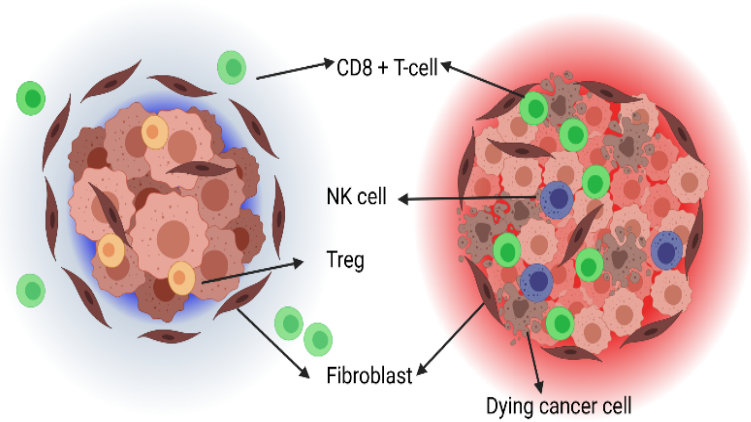

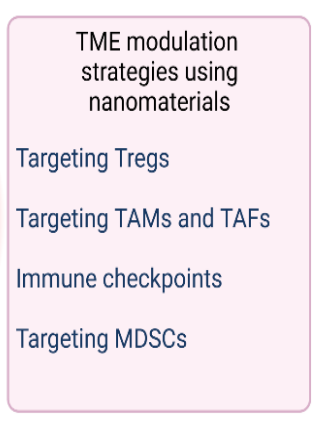

Figure 1. Schematic representation of (left) cold and (right) hot tumors depicting different cell types along with strategies applied while using nanomaterials for cancer immunotherapy (DC: dendritic cell, NK: natural killer cells, TAM: tumor associated macrophage, TAF: tumor associated fibroblasts, MDSC: myeloid-derived suppressor cells (Created using BioRender)

Research models have focused on engineering the tumor immune response and the use of checkpoint inhibitors and immune adjuvants have been beneficial in several cold tumor models which have a poor prognosis due to the presence of immunosuppressive cells that may be nonresponsive to immunotherapy ${ }^{35}$. Their mechanism of action is via binding to Toll like receptors (TLR), thereby enhancing recognition of poorly immunogenic antigens ${ }^{36}$. On the other hand, unwanted interactions with the immune system generate inflammatory or autoimmune disorders which may cause major therapeutic roadblocks. Although these pathways can be bypassed by suitable modification of nanomaterial properties, it does not completely suppress antibody production. Hence. there is a fine balance to maintain while using nanomedicine.

Cancer immunotherapy initially began with the injection of bacteria derived compounds which caused tumor shrinkage ${ }^{37}$ and paved the path for tuning and sensitizing the immune system. Ever since, several immunotherapy strategies exploiting neutrophils, macrophages, natural killer (NK) cells, T and B cells have been applied ${ }^{38-41}$. Nanomaterials have been used for immunotherapy to cure cancer due to several attributes which include long circulation times, protection of cargo from enzymatic degradation, delivery of antigens to antigen presenting cells (APCs) and regulation of the $\mathrm{TME}^{42}$. Immunotherapy using nanosystems can broadly be classified as immunosuppressive modulation of the TME and cancer nano vaccines. Nanoparticles can alter the TME without creating autoimmunity by selective and specific targeting to deliver therapeutics at the intended site. This is achieved by the enhanced permeability and retention (EPR) effect and using active and passive targeting. Nanocarriers have been used to regulate the TME by using immune checkpoints and mediators as well as targeting Tregs, tumor associated macrophages (TAMs) and fibroblasts (TAFs) and myeloid-derived suppressor cells (MDSCs) ${ }^{43}$. Codelivery of certain cytokines with small molecular inhibitors is a popular strategy in this regard.
For cancer vaccine development, commonly employed techniques include the simultaneous delivery of an antigen and an adjuvant, targeting dendritic cells (DCs), lymph node drainage, and antigen presentation. Antigens used for such vaccines can be either tumor-associated antigens (TAA), tumor-specific antigens (TSA), or cancertestis antigens (CTA). Combinatorial therapy employing traditional methods like radio and chemotherapy along with immunotherapy may also produce promising results in anti-cancer treatment.

As the first line of contact of nanomaterials in the body is with the immune system, of which the protein corona interaction is the most important, the surface properties of nanocarriers play a primordial role in determining their systemic fate. Coupled with insufficient knowledge of the interactions between these materials and ubiquitous biological components and tumor heterogeneity, it is impossible to envision a single system to suit all patients. Hence, there is an urgent need for personalized medicine considering several physiological and clinical factors for the best therapeutic outcome. Several efforts have been underway to address this issue including the use of modeling using CRISPR systems for the investigation of nanoparticle-mediated chemotherapy with TAM polarization ${ }^{44}$, evaluation of tumor biopsy including liquid biopsy using circulating tumor $\mathrm{DNA}^{45}$ and multi omics data analyses to identify the hallmarks and determining the molecular fingerprint of cancer ${ }^{46}$.

\section{References}

1. Harguindey S, Reshkin SJ. "The new pH-centric anticancer paradigm in Oncology and Medicine"; SCB, 2017. Seminars in Cancer Biology 2017, 43 (Supplement C), 1-4.

2. Gerweck LE, Seetharaman K. Cellular pH Gradient in Tumor $<$ em>versus</em> Normal Tissue: Potential Exploitation for the Treatment of Cancer. Cancer Research 1996, 56 (6), 1194.

3. Ray P, Kale N, Quadir M. New Side Chain Design for pH-Responsive Block Copolymers for Drug Delivery. Colloids and Surfaces B: Biointerfaces 2021, 111563. 
4. HuberV, Camisaschi C, Berzi A, et al. Cancer acidity: An ultimate frontier of tumor immune escape and a novel target of immunomodulation. Seminars in Cancer Biology 2017, 43 (Supplement C), 74-89.

5. Bailey KM, Wojtkowiak JW, Hashim AI, et al. Targeting the metabolic microenvironment of tumors. Adv Pharmacol 2012, 65, 63-107.

6. Confeld MI, Mamnoon B, Feng L, et al. Targeting the tumor core: hypoxia-responsive nanoparticles for delivery of chemotherapy to pancreatic tumors. Molecular Pharmaceutics 2020.

7. Evans ER, Bugga P, Asthana V, et al. Metallic nanoparticles for cancer immunotherapy. Materials Today 2018, 21 (6), 673-685.

8. Weinberg F, Ramnath N, Nagrath D. Reactive Oxygen Species in the Tumor Microenvironment: An Overview. Cancers (Basel) 2019, 11 (8), 1191.

9. Renoux B, Raes F, Legigan $\mathrm{T}$, et al. Targeting the tumour microenvironment with an enzyme-responsive drug delivery system for the efficient therapy of breast and pancreatic cancers. Chemical science 2017, 8 (5), 3427-3433.

10. Das A, Haque I, Ray P, et al. CCN5 activation by free or encapsulated EGCG is required to render triple-negative breast cancer cell viability and tumor progression. Pharmacol Res Perspect 2021, 9 (2), e00753.

11. Abdullah CS, Ray P, Alam S, et al. Chemical Architecture of Block Copolymers Differentially Abrogate Cardiotoxicity and Maintain the Anticancer Efficacy of Doxorubicin. Molecular Pharmaceutics 2020, 17 (12), 4676-4690.

12. Ray P, Ferraro M, Haag R, et al. Dendritic Polyglycerol-Derived NanoArchitectures as Delivery Platforms of Gemcitabine for Pancreatic Cancer. Macromol Biosci 2019, 19 (7), e1900073.

13. Sarker NC, Ray P, Pfau C, et al. Development of Functional Nanomaterials from Wheat Bran Derived Arabinoxylan for Nucleic Acid Delivery. Journal of Agricultural and Food Chemistry 2020, 68 (15), 4367-4373.

14. Ray P, Nair G, Ghosh A, et al. Microenvironment-sensing, nanocarriermediated delivery of combination chemotherapy for pancreatic cancer. Journal of cell communication and signaling 2019, 13 (3), 407420 .

15. Ray P, Confeld M, Borowicz P, et al. PEG-b-poly (carbonate)-derived nanocarrier platform with $\mathrm{pH}$-responsive properties for pancreatic cancer combination therapy. Colloids and Surfaces B: Biointerfaces 2019, 174, 126-135.

16. Ray P, Dutta D, Haque I, et al. pH-Sensitive Nanodrug Carriers for Codelivery of ERK Inhibitor and Gemcitabine Enhance the Inhibition of Tumor Growth in Pancreatic Cancer. Molecular Pharmaceutics 2021, 18 (1), 87-100.

17. Ray P, Alhalhooly L, Ghosh A, et al. Size-Transformable, Multifunctional Nanoparticles from Hyperbranched Polymers for EnvironmentSpecific Therapeutic Delivery. ACS Biomaterials Science \& Engineering 2019, 5 (3), 1354-1365.

18. Ray P, Gidley D, Badding JV, et al. UV and chemical modifications of polymer of Intrinsic Microporosity 1 to develop vibrational spectroscopic probes of surface chemistry and porosity. Microporous and Mesoporous Materials 2019, 277, 29-35.

19. Ray JK, Singha R, Ray D, et al. Palladium-catalyzed expedient Heck annulations in 1-bromo-1,5-dien-3-ols: Exceptional formation of fused bicycles. Tetrahedron Letters 2019, 60 (13), 931-935.

20. Ray $\mathrm{P}, \mathrm{Xu}$ E, Crespi VH, et al. In situ vibrational spectroscopy of adsorbed nitrogen in porous carbon materials. Physical Chemistry Chemical Physics 2018, 20 (22), 15411-15418.

21. Ray JK, Paul S, Ray P, et al. Pd-catalyzed intramolecular sequential Heck cyclization and oxidation reactions: a facile pathway for the synthesis of substituted cycloheptenone evaluated using computational studies. New Journal of Chemistry 2017, 41 (1), 278-284.
22. Chaudhuri S, Maity S, Roy M, et al. A Vinyl Radical Cyclization Route to Hydroxycyclohexene Fused Carbocycles. Asian Journal of Chemistry 2016, 28 (1).

23. Ray P, Gray JL, Badding JV, et al. High-Pressure Reactivity of Triptycene Probed by Raman Spectroscopy. The Journal of Physical Chemistry B 2016, 120 (42), 11035-11042.

24. Ray P. Interactions of nitrogen and hydrogen with various $1 \mathrm{D}$ and $3 \mathrm{D}$ carbon materials probed via in-situ vibrational spectroscopy. $P h . D$. Thesis 2016.

25. Wang C-Y, Ray P, Gong Q et al. Influence of gas packing and orientation on FTIR activity for $\mathrm{CO}$ chemisorption to the $\mathrm{Cu}$ paddlewheel. Physical Chemistry Chemical Physics 2015, 17 (40), 26766-26776.

26. Ghosh A, Sarkar S, Ghosh S, et al. Abstract 1234: Zoledronic acidinduced suppression of invasive phenotypes of pancreatic cancer cells is mediated through downregulation of CYR61/CCN1. Cancer research 2019, 79 (13 Supplement), 1234.

27. Brahma S, Ray P, Singha R, et al. Visible Colourimetric and Ratiometric Fluorescent Chemosensors for Cu (II) and Ni (II) Ions. Asian Journal of Chemistry 2016, 28 (5), 1035.

28. Singha R, Roy S, Nandi S, et al. Palladium-catalyzed one-pot SuzukiMiyaura cross coupling followed by oxidative lactonization: a novel and efficient route for the one-pot synthesis of benzo[c]chromene-6ones. Tetrahedron Letters 2013, 54 (7), 657-660.

29. Ray D, Nasima Y, Sajal MK, et al. Palladium-Catalyzed Intramolecular Oxidative Heck Cyclization and Its Application toward a Synthesis of $( \pm)$ - $\beta$-Cuparenone Derivatives Supported by Computational Studies. Synthesis 2013, 45 (09), 1261-1269.

30. Clément M, Abdellah I, Ray P, et al. Synthesis and NMR study of trimethylphosphine gold(i)-appended calix[8]arenes as precursors of gold nanoparticles. Inorganic Chemistry Frontiers 2020.

31. Ray P, Clément M, Martini C, et al. Stabilisation of small mono- and bimetallic gold-silver nanoparticles using calix[8]arene derivatives. New Journal of Chemistry 2018, 42 (17), 14128-14137.

32. André E, Boutonnet B, Charles P, et al. A New, Simple and Versatile Strategy for the Synthesis of Short Segments of Zigzag-Type Carbon Nanotubes. Chemistry - A European Journal 2016, 22 (9), 3105-3114.

33. Tran S, DeGiovanni P-J, Piel B, et al. Cancer nanomedicine: a review of recent success in drug delivery. Clin Transl Med 2017, 6 (1), 44-44.

34. Ray P, Haideri N, Haque I, et al. The Impact of Nanoparticles on the Immune System: A Gray Zone of Nanomedicine. Journal of Immunological Sciences 2021, 5 (1).

35. Jain P, Jain C, Velcheti V. Role of immune-checkpoint inhibitors in lung cancer. Therapeutic Advances in Respiratory Disease 2018, 12, 1753465817750075.

36. Shi S, Zhu H, Xia X, et al. Vaccine adjuvants: Understanding the structure and mechanism of adjuvanticity. Vaccine 2019, 37 (24), 3167-3178.

37. Andón FT, Digifico E, Maeda A, et al. Targeting tumor associated macrophages: The new challenge for nanomedicine. Semin Immunol 2017, 34, 103-113.

38. Gun SY, Lee SWL, Sieow JL, et al. Targeting immune cells for cancer therapy. Redox Biol 2019, 25, 101174-101174.

39. Leonard F, Curtis LT, Ware MJ, et al. Macrophage Polarization Contributes to the Anti-Tumoral Efficacy of Mesoporous Nanovectors Loaded with Albumin-Bound Paclitaxel. Front Immunol 2017, 8, 693.

40. Shi Y, Lammers T. Combining Nanomedicine and Immunotherapy. Accounts of chemical research 2019, 52 (6), 1543-1554.

41. Liu Z, Jiang W, Nam J, et al. Immunomodulating Nanomedicine for Cancer Therapy. Nano letters 2018, 18 (11), 6655-6659. 
42. Qian H, Liu B, Jiang X. Application of nanomaterials in cancer immunotherapy. Materials Today Chemistry 2018, 7, 53-64.

43. Song W, Musetti SN, Huang L. Nanomaterials for cancer immunotherapy. Biomaterials 2017, 148, 16-30.

44. Leonard F, Curtis LT, Hamed AR, et al. Nonlinear response to cancer nanotherapy due to macrophage interactions revealed by mathematical modeling and evaluated in a murine model via
CRISPR-modulated macrophage polarization. Cancer Immunology, Immunotherapy 2020, 69 (5), 731-744.

45. Barlebo Ahlborn L, Østrup 0. Toward liquid biopsies in cancer treatment: application of circulating tumor DNA. Apmis 2019, 127 (5), 329-336.

46. Vucic EA, Thu KL, Robison K, et al. Translating cancer 'omics' to improved outcomes. Genome Res 2012, 22 (2), 188-195. 